\title{
$\underline{\mathbf{P}-159}$
}

\section{Identification of Alkaloid compounds and Antioxidant Activity of Rafflesia Cantleyi and Its Host, Tetrastigma Tuberculatum}

\author{
Muhammad Azhar Z, Zaini Y and Nor Shuhada O* \\ Faculty of Applied Science, Universiti Teknologi MARA Perlis, 02600 Arau, Perlis, Malaysia; \\ E-mail:da_imie@yahoo.com
}

Rafflesia cantleyi is a parasitic flowering plant which belongs to Rafflesiaceae family. The parasitic plant grows on Tetrastigma tuberculatum, a member of Vitaceae family. Also known as obligate holoparasite, Rafflesia cantleyi can be found in penisular Malaysia as well as Borneo but in rare and limited distributions. In this study, the alkaloid compounds in the flower bud of Rafflesia cantleyi and the roots of its host, Tetrastigma tuberculatum are identified using phytochemical screening method and HPLC technique. The sample of Rafflesia cantleyi and Tetrastigma tuberculatum were collected from Belum Valley, Banding Lake, Perak. Air dried powdered samples were first extracted using dichloromethane followed by methanol. The methanol extract of R.cantleyi and T.tuberculatum were then subjected to alkaloid screening test ${ }^{2}$. The results showed strong positive test for T.tuberculatum indicating the presence of alkaloid compounds in T.tuberculatum. However, the screening test for R.cantleyi showed negative result for alkaloid content. Both extract were then subjected to HPLC for alkaloid identification using caffeine as standard. Based on the HPLC spectrum obtained, caffeine is presence in both R.cantleyi and T.tuberculatum. The methanol extract of both samples were also tested for antioxidant activity by using DPPH free radical scavenging activity ${ }^{3}$. Result from the DPPH method showed that the radical scavenging activity of R.cantleyi is $96.0 \%$ whereas for T.tuberculatum is $97.0 \%$ which is quiet high when compared to standard alpha tocopherol (natural antioxidant) and BHT (synthetic antioxidant) which have the value of $97.0 \%$ and $96.0 \%$ antioxidant activities respectively. 